\title{
Perinodal slow potential as a local guide for transcatheter radiofrequency ablation of atrioventricular nodal reentrant tachycardia: therapeutic efficacy and electrophysiological mechanisms of success
} Jiunn-Lee Lin, Fang-Yue Lin, Huey-Ming Lo, Chuen-Den Tseng, Tin-Fu Cheng,
Jin-Jer Chen, Yung-Zu Tseng, Wen-Pin Lien

Division of

Cardiology

Department of

Internal Medicine,

National Taiwan

University Hospital,

Taipei, Taiwan,

Republic of China

$\mathrm{J}-\mathrm{L}$ Lin

C-D Tseng

J-J Chen

Y-Z Tseng

W-P Lien

Division of

Cardiovascular

Surgery, Department

of Surgery, National

Taiwan University

Hospital, Taipei,

Taiwan, Republic of

China

F-Y Lin

Department of

Medicine, Provincial

Taipei Hospital,

Taipei, Taiwan,

Republic of China

T-F Cheng

Department of

Medicine, Provincial

Tao-Yuan Hospital,

Tao-Yuan, Republic of

China

H-M Lo

Correspondence to:

Dr W-P Lien, Division of

Cardiology, Department of

Internal Medicine, National

Taiwan University Hospital,

No 7 Chun-Shan South

Road, Taipei, Taiwan 100,

Republic of China.

Accepted for publication

13 March 1995

\section{Abstract}

Background-A specific local indicator in the Koch's triangle could be critical to the complication-free treatment of atrioventricular nodal reentrant tachycardia by transcatheter radiofrequency ablation. Recording of perinodal slow potential reflects a slow conduction area, and probably indicates the location of the slow pathway component of the circuit. Specific ablation of the slow pathway would carry the least risk of atrioventricular block.

Methods and results-Guided by the mapped perinodal slow potential, atrioventricular nodal reentrant tachycardia was successfully eliminated in all of 55 consecutive patients in one session. Fifty two patients $(94 \cdot 5 \%)$ had confirmed slow potential at the final success sites. Despite the good result, the underlying electrophysiological mechanisms of early success from slow-potential-guiding catheter ablation were heterogeneous: selective slow pathway eradication in 31 patients (56.4\%, group A), selective slow pathway modification in 18 patients (32.7\%, group B), inadvertent fast pathway damage in six patients $(10.9 \%$, group C). Group B patients had the preservation of dual atrioventricular nodal pathways, adequate atrio-Hisian delay, fast pathway facilitation, and a higher frequency of inducible, single non-conducted nodal echo $(15 / 18,83.3 \% v 6 / 31$, $19.4 \%$ in group $A, P \ll 0.001)$. The upper communicating path of the circuit was implicated as another site of radiofrequency destruction. Three recurrences were documented in follow up study. However, reablation by the same approach caused complete atrioventricular block in one patient $(1 \cdot 7 \%, 1 / 58$ procedures). None of the local characteristics of ablation sites was an independent predictor of procedure outcome.

Conclusions-Perinodal slow potential is not a specific slow pathway indicator in transcatheter radiofrequency ablation of atrioventricular nodal reentrant tachycardia. Multiple strategic sites of the reentry circuit may be damaged through similar local signals.
Keywords: perinodal slow potential; reentrant tachycardia; transcatheter radiofrequency ablation

\section{(Br Heart f 1995;74:268-276)}

The recent advance in the definitive treatment of the atrioventricular nodal reentrant tachycardia is astonishing, given the persistent controversy in the exact tissue components of the reentry circuit. With the present knowledge of local anatomy and traditional electrophysiological study, both surgery ${ }^{12}$ and catheter ablation ${ }^{34}$ have been shown to be successful in eliminating reentrant tachycardia by selective damage of either the anterograde slow pathway or the retrograde fast pathway. However, the lack of a specific electrical marker for a destructive procedure in the critical atrioventricular junctional area has always been a drawback in the achievement of a successful result without major complication, that is, high grade atrioventricular block.

Perinodal slow potential is a local signal recordable from Koch's triangle of the atrioventricular junctional area. The small low frequency local potential with decremental conduction property was first studied for the purpose of therapeutic catheter ablation by Haissaguerre et al in $1992 .{ }^{4} \mathrm{He}$ showed the slow potential to be a specific electrical marker for complication-free slow pathway ablation of atrioventricular nodal reentrant tachycardia. Later, direct contact mappings ${ }^{5-7}$ by high density, high resolution plaque electrodes have also confirmed the existence of similar slow potentials located between the atrioventricular node and the coronary sinus ostium in canine, porcine, and human hearts. However, the histological, functional, and clinical correlations of the slow potential to the atrioventricular nodal reentrant tachycardia are still speculative, and by no means conclusive.

With the recognition of the importance of perinodal atrial tissue ${ }^{89}$ and the relevant conduction slowing in atrioventricular nodal reentrant tachycardia, ${ }^{3-5}$ we hypothesised the recording of the slow potential in the Koch's triangle as a reasonable indicator for the slow pathway component of the reentry circuit. The present prospective study was thus conducted to evaluate the therapeutic effect, and the underlying electrophysiological mechanisms of the functional outcome, of 
transcatheter radiofrequency ablation of atrioventricular nodal reentrant tachycardia guided by the perinodal slow potential, so as to elucidate the clinical implications of the local electrical landmark in the atrioventricular junction.

\section{Methods \\ PATIENTS}

Between May 1992 and October 1993, 55 consecutive patients with paroxysmal supraventricular tachycardia, which then proved to be slow-fast atrioventricular nodal reentrant tachycardia, were referred for transcatheter radiofrequency ablation treatment. They were 17 men and 38 women. Their age ranged from 16 to 70 years, mean 53 (SD 8) years. Mean duration of clinical recurrent tachycardia was 8.0 (3.0) years. Coexistent cardiovascular diseases were rheumatic valvular disease in two patients (mitral stenosis after percutaneous mitral valvuloplasty in one; mitral regurgitation in one), congenital heart disease in two patients (secundum atrial septal defect in one; perimembranous ventricular septal defect in one), coronary artery disease in one patient, and combined atrioventricular reentrant tachycardia incorporating concealed left posterior accessory atrioventricular pathway in one patient. Two patients had had previous fast pathway modification, and the recurrence of slow-fast atrioventricular nodal reentrant tachycardia. One of the latter two patients had first degree atrioventricular block.

\section{BASELINE ELECTROPHYSIOLOGY STUDY}

Before transcatheter radiofrequency ablation, every patient underwent a complete electrophysiological study, which was performed in a postabsorptive, non-sedated state. Informed written consent was obtained before the study. Our investigational protocol was approved by the hospital research committee, and was in compliance with local ethics standards. During the study, three $6 \mathrm{~F}$ quadripolar electrode catheters with $1 \mathrm{~cm}$ interelectrode distance (USCI, or Mansfield-Webster) were introduced through femoral veins, advanced into the right atrium, then positioned at high right atrium, His bundle region, and right ventricular apex. A fourth $7 \mathrm{~F}$ decapolar electrode catheter (USCI, interelectrode distance of $0.5 \mathrm{~cm}$ ) or orthogonal electrode catheter with three groups of circumferentially arranged electrode pieces (Mansfield/ Webster, interelectrode distance of $1 \mathrm{~mm}$ between orthogonal pieces) was inserted through the jugular vein and positioned in the coronary sinus. The proximal pair or group of this catheter was carefully positioned in close proximity to the coronary sinus ostium. Surface electrocardiogram leads $\left(\mathrm{I}, \mathrm{aVF}, \mathrm{V}_{1}\right)$, as well as intracardiac local electrograms from multiple sites, were simultaneously displayed and recorded on a multichannel oscilloscope recorder (EVR-110, PPG Biomedical) at a paper speed of 100 or $150 \mathrm{~mm} / \mathrm{s}$. The electrical stimulation was performed with a digital pro- grammable stimulator (Bloom Associates). Pacing stimuli were twice the diastolic threshold in strength and $2 \mathrm{~ms}$ in duration. The stimulation protocol encompassed incremental atrial and ventricular pacing, and extrastimulation coupling on two basic driving cycle lengths (usually $600 \mathrm{~ms}$ or 500 $\mathrm{ms}$, and $400 \mathrm{~ms}$ ). The induction of atrioventricular nodal reentrant tachycardia was attempted repeatedly to find out the most reliable and reproducible protocol of tachycardia initiation. If the atrioventricular nodal reentrant tachycardia could not be induced or sustained in the baseline state, isoprenaline was infused and titrated to an increase of at least $20 \%$ of the heart rate. The same pacing protocol was then repeated. With such study protocol, all 55 patients were shown to have dual atrioventricular nodal pathways with the demonstration of discontinuous atrioventricular nodal conduction curve through atrial extra-stimulation (50 patients), incremental atrial pacing (39 patients), ventricular extrastimulation (15 patients), and incremental ventricular pacing (seven patients). All 55 patients in the present study had reproducible sustained atrioventricular nodal reentrant tachycardia by the stimulation protocol. Twenty two of the 55 patients required isoprenaline infusion to facilitate the tachycardia induction.

PERINODAL SLOW POTENTIAL RECORDING

Our working hypothesis for effective slow pathway ablation assumed the perinodal slow potential to be an electrical landmark of the critical slow conduction area, that is, the atrioventricular nodal slow pathway, of the reentry circuit. Systematic mapping was conducted by a deflectible, large tip ( $4 \mathrm{~mm}$ ) quadripolar catheter with 2-5-2 mm interelectrode distance (Mansfield/Webster) along the anterior, median, and posterior septal zones of the tricuspid annular border of Koch's triangle. Bipolar local electrogram recorded from the distal electrode pair was magnified at $0.05-0.2 \mathrm{mV} / \mathrm{cm}$ with a filter range of 30 and $500 \mathrm{~Hz}$. Any focus manifesting multiphasic atrial electrogram or distinct interpolated low frequency potential was selected and verified by low rate atrial overdriving, usually $20-50 \%$ above the sinus rate. If a rounded or biphasic small potential was dissociable from the local atrial electrogram and diminished in amplitude with the gradual increase of atrial pace rate, the selected site was confirmed to have the perinodal slow potential (fig 1 ). If pacing verification was impossible due to catheter instability or too low signal amplitude, the site with a suspicious slow potential was still picked up as an ablation target, when there was no better choice. Since the slow potentials were frequently recorded at two or even three contiguous zones between the His bundle position and the coronary sinus ostium, the transcatheter radiofrequency ablation was usually targeted first to the most posteromedial zone. Local His potential, atrial repolarisation and local artefacts were excluded by the following properties of the 


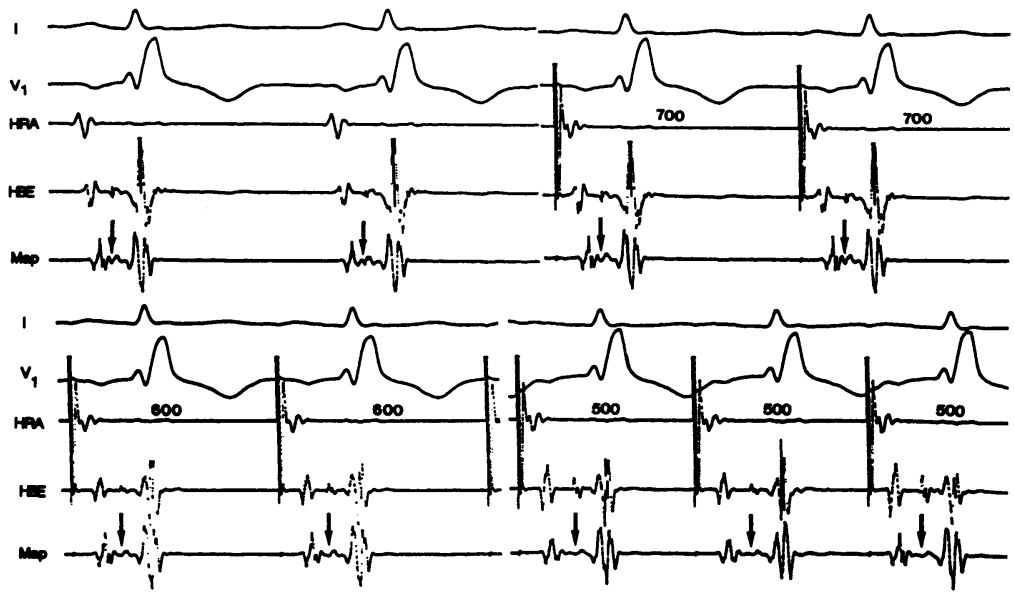

Figure 1 Recording and pacing verification of perinodal slow potential. The low frequency biphasic potential (arrow) is interposed between the local atrial and ventricular electrograms. The slow potential could be dissociated gradually from the atrial component and merged towards the ventricular component with a decrease in right atrial pacing cycle length from $700 \mathrm{~ms}$ to $600 \mathrm{~ms}$ and $500 \mathrm{~ms}$, as shown in the four panels. For each panel, the tracings include surface electrocardiograms, $I, V_{1}$, intracardiac electrograms at high right atrium (HRA), His bundle (HBE), and the mapping site (Map)

slow potential: (1) rate dependent gradual migration and convergence of slow potential into local ventriculogram; (2) fixed His-ventricular interval over a range of atrial pacing cycle lengths; (3) relative constancy of the local activation time of the slow potential; (4) specific anatomical distribution of the slow potential.

\section{CATHETER ABLATION}

After the selection of an appropriate target site by the above local electrographic criteria, radiofrequency energy of $500 \mathrm{kHz}$ was transmitted between the $4 \mathrm{~mm}$ bulbous tip ( 27 $\mathrm{mm}^{2}$ surface area, Mansfield/Webster) of the ablation catheter and a dispersive patch electrode on the left posterior chest wall. The energy was supplied by a commercially available generator (Radionics $3 \mathrm{C}$ ) in a continuous unmodulated sine wave mode. Power, voltage, current, local impedance, and timing were displayed on the monitor screen of the generator machine but the data were not stored. For each ablation attempt, the power setting was always started from 10-15 W and gradually increased to $20-30 \mathrm{~W}$, each for $30 \mathrm{~s}$ duration. The surface electrocardiogram was closely monitored. In case of appearance of junctional tachycardia during energy delivery, atrial overdrive pacing was applied to guarantee a better observation of atrioventricular conduction. After each ablation, tachycardia inducibility was immediately retested by similar stimulation protocol as in the baseline study. The therapeutic end point was the noninducibility of atrioventricular nodal reentrant tachycardia and no more than single non-conducted echo beat using the programmed atrial and ventricular stimulation protocol with isoprenaline facilitation.

\section{FOLLOW UP}

After the laboratory success, all 55 patients were followed regularly in the outpatient clinic or by the local referring doctor for at least six months (mean 18 (5) months, range 9 to 27 months). On monthly follow up visits, detailed history taking of any symptomatic recurrence of tachycardia, physical examination, 12-lead surface electrocardiogram, and occasional 24 hour Holter electrocardiogram study were carefully evaluated. After explanation, $40(72 \cdot 7 \%)$ of the 55 patients accepted laboratory electrophysiological restudy 3-4 months after the initial procedure.

\section{ELECTROPHYSIOLOGIC DATA ANALYSIS}

Before the catheter ablation, the diagnosis of atrioventricular nodal reentrant tachycardia was established according to the criteria suggested by many investigators ${ }^{1011}$ : (1) demonstration of discontinuous atrioventricular nodal conduction curve, and the induction of tachycardia when the critical atrio-Hisian interval is achieved; (2) atrial activation simultaneous with, or earlier than, ventricular activation during tachycardia; (3) no advancement of atrial activation by ventricular extrastimulation introduced when $\mathrm{His}$ bundle was refractory; (4) identical septal sequence of retrograde atrial activation during tachycardia and ventricular pacing; (5) constant His-atrial interval of the return cycle after an introduced atrial extrastimulation over a wide range of coupling intervals during tachycardia. Criteria 2 and 3 excluded the incorporation of an accessory atrioventricular pathway. Criteria 4 and 5 excluded the possibility of atrial tachycardia.

In order to evaluate the underlying electrophysiological mechanism, the atrioventricular nodal function was carefully compared between the electrophysiological studies performed before, early after (30 minutes to one hour), and late (three to four months) after the transcatheter radiofrequency ablation. The electrophysiological data included the basic sinus cycle length, the atrio-Hisian interval, the atrioventricular nodal effective refractory period, the atrioventricular nodal blocking cycle length, 1:1 conducting cycle length of the fast and slow pathways, the effective refractory periods of the fast and slow pathways, the atrial effective refractory period, and the inducibility of atrioventricular nodal reentrant echoes or tachycardia. The effective refractory periods were compared at the same, or the closest possible driving cycle length in the baseline state or the same autonomic stress state, or both, by single atrial extrastimulation technique. Atrioventricular nodal conduction curve was depicted, classified, and compared among the three study phases using incremental atrial pacing or atrial extrastimulation technique.

For the recorded perinodal slow potential, the following characteristics were analysed: local atrio-slow potential interval, normalised slow potential activation time, slow potential amplitude, and the anatomical location of the slow potential in Koch's triangle. Classically, the His bundle recording area (bipolar signal, $1 \mathrm{~cm}$ interpolar distance) represented the anterior atrionodal input zone of the normal 
Figure 2 Normalised slow potential activation time. The slow potential activation time relative to the normal atrioventricular conduction is estimated by the ratio of corrected local atrio-slow potential interval versus atrio-

Hisian interval of $\mathrm{Hi}$ bundle electrogram $(H B E)$, that is, $c(A-S P) / A-H$. $H, H i s$ bundle. Other

abbreviations as in fig 1. Time scale is shown at the bottom.
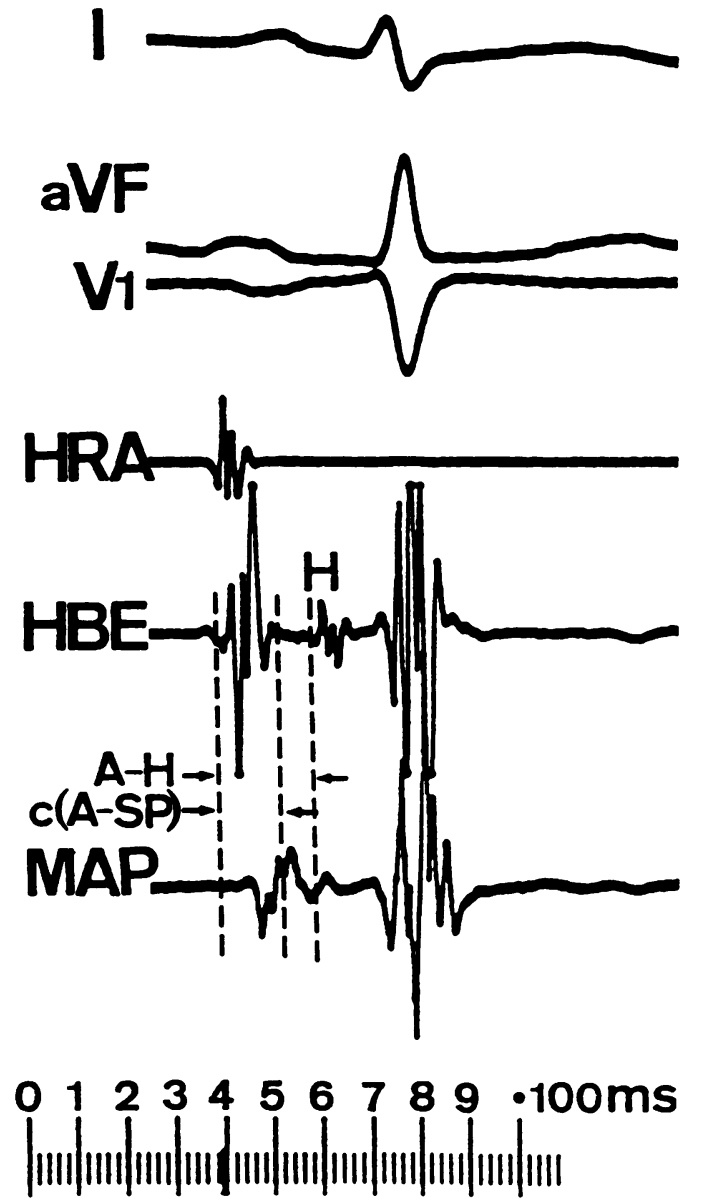

atrioventricular conduction in human. The relative timing of the slow potential activation as normalised to the atrio-Hisian interval (fig 2) was to highlight the relative activation sequence of the slow potential in normal atrioventricular conduction.

\section{STATISTICS}

Basic comparative statistics was calculated by the paired Student $t$ test for dependent continuous data, and the $\chi^{2}$ test with Yates correction for categoric data. All continuous data are expressed as mean (SD). A probability value of less than 0.05 was considered significant.
In order to understand the contribution of perinodal slow potential to the successful catheter ablation of atrioventricular nodal reentrant tachycardia, univariate followed by multivariate logistic regression analysis was applied to extract the independent factors predicting procedure outcome, by means of the Statistical Analysis System (SAS) program. Sensitivity, specificity, positive and negative predictive values, and predictive accuracy were calculated for the selected predictors, if any.

\section{Results \\ PERINODAL SLOW POTENTIAL AND CATHETER \\ ABLATION}

Along the tricuspid border of Koch's triangle, meticulous mapping followed by pacing verification confirmed the existence of definite perinodal slow potential at the successful ablation sites in 52 of the 55 patients $(94.5 \%)$. However, all 55 patients had had successful elimination of the slow-fast atrioventricular nodal reentrant tachycardia in one single procedure. The lack of a definite slow potential did not prohibit the successful catheter ablation in the remained three patients, who had only a suspicious local signal. Mean radiofrequency pulse number required for the successful outcome was $2 \cdot 4(2 \cdot 9)$ (range 1 to 20 ). Mean total procedure time was $2 \cdot 8(1 \cdot 1)$ hours. Mean fluoroscopic time allocated to mapping and ablation was 23.4 (14.9) minutes, ranging from 50-60 minutes in the learning period to $10-15$ minutes in the latter half of the series. The confirmed slow potentials could be recorded from one, two, or even all three zones in the Koch's triangle: the posteroseptal zone alone in three patients, the medioseptal zone alone in 32 patients, the anteroseptal zone alone in one patient, the medioseptal and posteroseptal zones in 13 patients, and all three zones in three patients. The most posteromedial site was selected for the first ablation.

The mere recording of perinodal slow potential in the critical triangle of Koch did not guarantee the success of transcatheter radiofrequency ablation of atrioventricular nodal reentrant tachycardia. Analysis of local electrograms of the selected targets of the first

Table 1 Local electrogram characteristics of the successful and failed target sites of the first and overall ablation attempts. Values are means (SD)

\begin{tabular}{|c|c|c|c|c|c|c|}
\hline & \multicolumn{3}{|l|}{ First ablation site } & \multicolumn{3}{|l|}{ All ablation sites } \\
\hline & Success & Failure & $P \leqslant$ & Success & Failure & $P \leqslant$ \\
\hline $\begin{array}{l}\text { PNSP (+) } \\
\text { A-SP (ms) } \\
\text { c(A-SP)/A-H (\%) } \\
\text { PNSP amplitude (mV) } \\
\text { Koch's triangle zone: }\end{array}$ & $\begin{array}{l}31 / 32(96 \cdot 9 \%) \\
50 \cdot 8(10 \cdot 3) \\
73 \cdot 5(19 \cdot 2) \\
0.041(0.022)\end{array}$ & $\begin{array}{l}17 / 23(73.9 \%) \\
54 \cdot 4(11 \cdot 6) \\
87.5(22 \cdot 0) \\
0.036(0.020)\end{array}$ & $\begin{array}{l}0.012 \\
\text { NS } \\
0.024 \\
\text { NS } \\
0.012\end{array}$ & $\begin{array}{l}52 / 55(94.5 \%) \\
50 \cdot 6(10 \cdot 2) \\
75 \cdot 8(18 \cdot 9) \\
0.040(0.025)\end{array}$ & $\begin{array}{l}37 / 71(52 \cdot 1 \%) \\
53 \cdot 0(9 \cdot 3) \\
86 \cdot 5(18 \cdot 7) \\
0.031(0 \cdot 016)\end{array}$ & $\begin{array}{l}0.00000 \\
\text { NS } \\
0.0095 \\
0.054 \\
0.001\end{array}$ \\
\hline $\begin{array}{l}\text { posteroseptal } \\
\text { medioseptal } \\
\text { anteroseptal }\end{array}$ & $\begin{array}{l}6 \\
25 \\
1 \\
26 / 32(81.3 \%)\end{array}$ & $\begin{array}{l}13 \\
10 \\
0 \\
12 / 23(52.2 \%)\end{array}$ & & $\begin{array}{l}9 \\
43 \\
3 \\
42 / 55(76.4 \%)\end{array}$ & $\begin{array}{l}34 \\
34 \\
3 \\
23 / 71(32.4 \%)\end{array}$ & \\
\hline Heat JT (+) & $26 / 32(81 \cdot 3 \%)$ & $12 / 23(52 \cdot 2 \%)$ & 0.021 & $42 / 55(76 \cdot 4 \%)$ & $23 / 71(32 \cdot 4 \%)$ & 0.00000 \\
\hline
\end{tabular}

PNSP (+), with perinodal slow potential; A-SP, local atrio-slow potential interval; c(A-SP), corrected local atrio-slow potential interval; A-H, atrio-Hisian interval at His bundle electrogram; Koch's triangle zone, specific location of the ablation site at the tricuspid border of the Koch's triangle; Heat $J T(+)$, with junctional tachycardia during radiofrequency ablation. 
and overall ablation attempts revealed only $64.6 \%(31 / 48)$ and $58.4 \%(52 / 89)$ of the slow potential recording sites had subsequently resulted in the successful elimination of the tachycardia. Other than the existence of perinodal slow potential, the success sites by single radiofrequency pulse usually had several other characteristics (table 1): (1) corrected activation time of the slow potential corresponded to $73.5(19.2) \%$ (range $33.3 \%$ to $113 \%$ ) of the atrio-Hisian interval on the His bundle electrogram (versus $87.5(22.0) \%$, range $50 \%-142 \%$, at failure sites, $P \leqslant 0.024$ ); (2) more frequent localisation at the medioseptal zone of Koch's triangle (78.1\%, versus $43.5 \%$ at failure sites, $P \leqslant 0.012$ by $\chi^{2}$ ); (3) more frequent emergency of junctional tachycardia during radiofrequency energy delivery $(81.3 \%$, versus $52.2 \%$ at failure sites, $\mathrm{P} \leqslant$ 0.021 ). Accumulated analysis of the local electrograms of overall ablation attempts showed the same trend (table 1).

However, further multivariate analysis was unable to isolate any local characteristic independently predicting the procedure outcome of the first ablation attempt in the 55 patients. Only when neglecting the possible effect of accumulated tissue damage and pooling the data from all ablation attempts did the recording of perinodal slow potential and heating junctional tachycardia emerge as independent predictors of success. However, the positive predictive value and the predictive accuracy remained inadequate: $58.4 \%$ and $68.3 \%$ for perinodal slow potential alone, $64.2 \%$ and $71.4 \%$ for junctional tachycardia alone, $69.4 \%$ and $75.4 \%$ for the two together.
ELECTROPHYSIOLOGICAL MECHANISMS OF EARLY SUCCESS

After the procedure success, three main mechanisms of atrioventricular nodal modification were found by repeated electrophysiological study, and recognised as responsible for the non-inducibility of the clinical tachycardia. The three electrophysiological mechanisms elucidated by the atrioventricular nodal conduction patterns (fig 3 ) were the selective eradication of the slow pathway, the selective modification of the slow pathway, and the selective modification of the fast pathway.

\section{Selective slow pathway eradication}

In 31 of the 55 patients $(56.4 \%)$ (group A) the discontinuous atrioventricular nodal conduction curve disappeared (A1, A2 in fig 3) early after the success of transcatheter radiofrequency ablation, suggesting the eradication of the slow pathway. Overall, the 31 patients had shortening of the sinus cycle length, the 1:1 conducting cycle length of the fast pathway, and the effective refractory period of the fast pathway after the ablation (table 2). None of the 31 patients had sustained 1:1 conduction of the slow pathway. Maximum atrio-Hisian interval obtainable after ablation was less than the minimum requirement of the atrio-Hisian delay for the induction of the reentrant tachycardia. Among the 31 patients, 13 were shown to have premature interruption of the atrioventricular nodal conduction (subgroup A1) (A1 in fig 3), that is, prolongation of the atrioventricular nodal effective refractory period. The other 18 patients had only the "smoothing" of
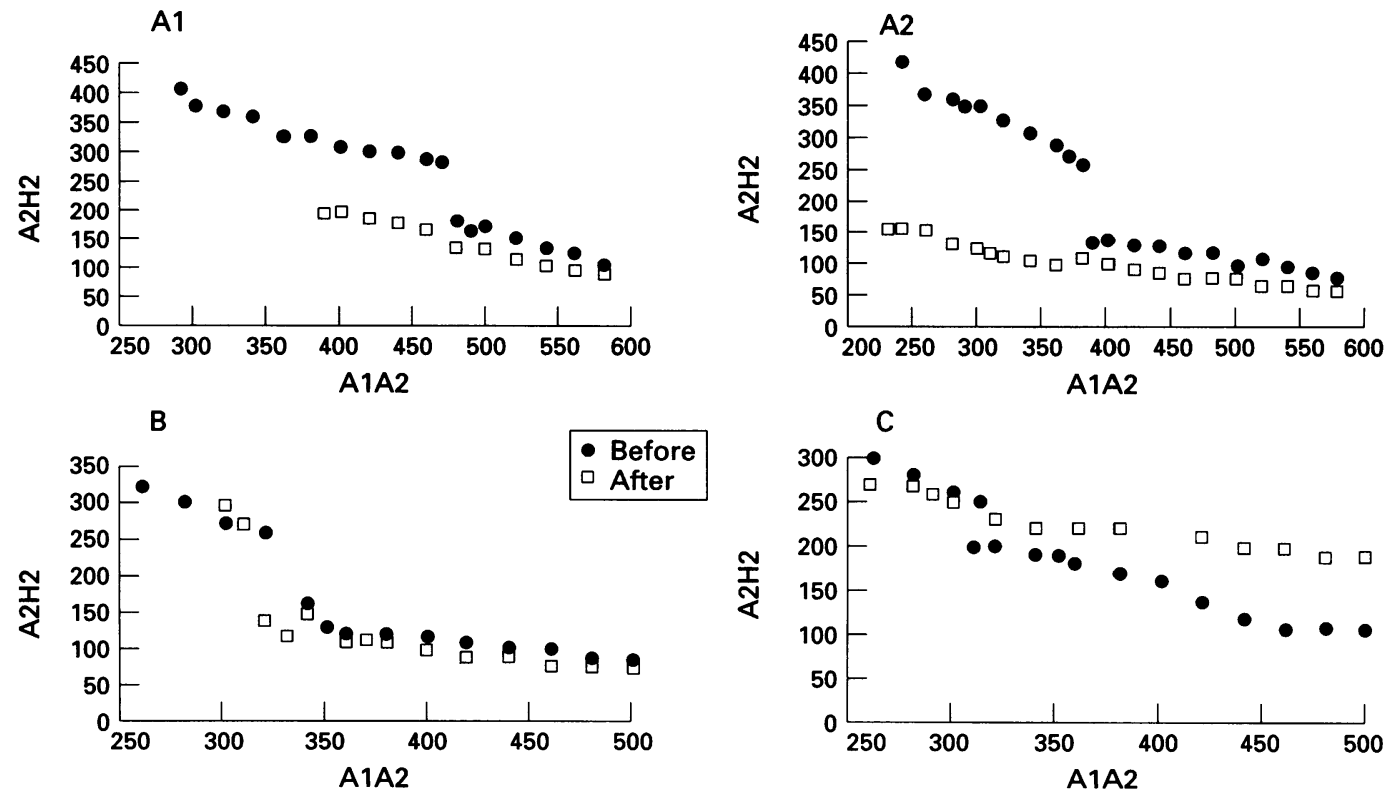

Figure 3 Comparison of atrioventricular nodal conduction curves before and after the success of transcatheter radiofrequency ablation of atrioventricular nodal reentrant tachycardia. Each panel shows the paired data of atrio-Hisian intervals (A2H2) and atrial coupling intervals (A1A2) derived from single atrial extrastimulus study. The four panels represent examples of selective slow pathway eradication (panels $A 1, A 2$ ), selective slow pathway modification and upper communicating path damage (panel B), and fast pathway damage (panel C). Panels A1 and A2 show the difference of atrioventricular nodal refractoriness between the two corresponded subgroups of patients, despite similar disappearance of the slow pathway behaviour after ablation. Panel B shows the postablation preservation of the dual atrioventricular nodal pathways. The effective refractory period of the residual slow pathway is prolonged after ablation in this particular group $B$ patient, whereas it is unchanged or shortened in many others from the same group. Panel $C$ shows the all slow pathway behaviour with the inadvertent damage of the antegrade fast pathway. Intervals of $A 2 H 2$ and A1A2 in ms. 
Table 2 Electrophysiological characteristics of the 31 group $A$ patients before and soon after the catheter ablation. Values are means (SD), ms

\begin{tabular}{|c|c|c|c|c|c|c|}
\hline & \multicolumn{3}{|c|}{ Subgroup $A 1(n=13)$} & \multicolumn{3}{|c|}{ Subgroup $A 2(n=18)$} \\
\hline & Before & After & $P \leqslant$ & Before & After & $P \leqslant$ \\
\hline $\begin{array}{l}\text { Sinus CL } \\
\text { A-H } \\
\text { Max }(A-H) \\
\text { Min }(A-H)_{\text {svT }} \\
\text { FPERP } \\
\text { SPERP } \\
\triangle \text { ERP } \\
1: 1 \text { FP } \\
1: 1 \text { SP } \\
1: 1 \text { VA }\end{array}$ & $\begin{array}{c}800 \cdot 8(111 \cdot 2) \\
73 \cdot 4(15 \cdot 2) \\
370 \cdot 0(73 \cdot 8) \\
261.5(72 \cdot 3) \\
387 \cdot 7(101 \cdot 2) \\
261 \cdot 5(36 \cdot 3) \\
126 \cdot 2(89 \cdot 6) \\
476 \cdot 2(112 \cdot 1) \\
367 \cdot 8(53 \cdot 8) \\
413 \cdot 1(80 \cdot 4)\end{array}$ & $\begin{array}{l}727 \cdot 2(119 \cdot 2) \\
73.9(16 \cdot 1) \\
176 \cdot 2(37 \cdot 3) \\
\overline{336} \cdot 2(42 \cdot 5) \\
= \\
\overline{396} 6 \cdot 2(69 \cdot 6) \\
\overline{35} 3.9(69.5)\end{array}$ & $\begin{array}{l}0.070 \\
\text { NS } \\
0.00002 \\
- \\
0.025 \\
- \\
0.002 \\
\overline{0} \\
0.043\end{array}$ & $\begin{array}{r}725 \cdot 0(113.4) \\
78.9(38.3) \\
360.0(89.3) \\
288.3(91.4) \\
296 \cdot 1(47 \cdot 2) \\
238.8(35.6) \\
58 \cdot 1(37.6) \\
375.6(76.4) \\
356.7(75 \cdot 7) \\
352.2(84.1)\end{array}$ & $\begin{array}{l}661 \cdot 1(103 \cdot 1) \\
76 \cdot 1(34 \cdot 2) \\
201 \cdot 6(62 \cdot 5) \\
\frac{2}{224} \cdot 4(47 \cdot 6) \\
\frac{32}{32} \cdot 4(60 \cdot 6) \\
332 \cdot 2(49 \cdot 3)\end{array}$ & $\begin{array}{l}0.001 \\
\text { NS } \\
0.00001 \\
\overline{0.00009} \\
\overline{-} \\
\overline{0.002} \\
\overline{\text { NS }}\end{array}$ \\
\hline
\end{tabular}

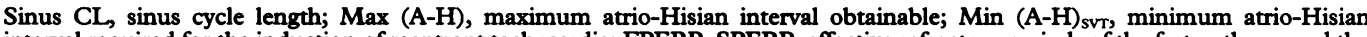
interval required for the induction of reentrant tachycardia; FPERP, SPERP, effective refractory periods of the fast pathway and the slow pathway, $\triangle E R P$, difference of the effective refractory periods of the fast and the slow pathways; 1:1 FP, 1:1 SP, minimum 1:1 conducting cycle lengths of the fast pathway and the slow pathway; 1:1 VA, minimum 1:1 conducting cycle length of retrograde ventriculo-atrial conduction. $P$ value, comparison of data before and early after catheter ablation.

the atrioventricular nodal function curve (subgroup A2) (A2 in fig 3), that is, the same or shorter atrioventricular nodal effective refractory period. The frequency of induction of single, non-conducted slow-fast atrioventricular nodal reentrant echo was rather greater in patients of subgroup A2 (5/18, $27.8 \%$ ) than those of subgroup A1 $(1 / 13$, $7 \cdot 7 \%, P>0.05)$. The subgroup A1 (13 patients, $23.6 \%$ of all 55 patients) might represent those who had more definite eradication of the slow pathway of the atrioventricular nodal reentrant circuit early after the success.

\section{Selective slow pathway modification}

Eighteen (32.7\%) (group B) of the 55 patients had the persistence of the atrioventricular nodal dual pathways after the successful catheter ablation (B in fig 3). Electrophysiologically, they had also had the shortening of the sinus cycle length, the $1: 1$ conducting cycle length of the fast pathway, and the effective refractory period of the fast pathway (table 3). None of the 18 patients had sustained 1:1 conduction of the slow pathway either. However, the duality of the atrioventricular nodal conduction persisted. The effective refractory period of the slow pathway determined by extrastimulus study was little changed after the catheter ablation. The maximum atrio-Hisian interval obtainable remained more than that needed for the induction of the tachycardia. The frequency of the induction of single, non-conducted

Table 3 Electrophysiological characteristics of the 18 group $B$ patients before, and soon after the catheter ablation. Values are means (SD), ms

\begin{tabular}{|c|c|c|c|}
\hline & Before & After & $P \leqslant$ \\
\hline $\begin{array}{l}\text { Sinus CL } \\
\text { A-H } \\
\text { Max }(A-H) \\
\text { Min }(A-H)_{\text {svT }} \\
\text { FPERP } \\
\text { SPERP } \\
\triangle \text { ERP } \\
1: 1 \text { FP } \\
1: 1 \text { SP } \\
1: 1 \text { VA }\end{array}$ & $\begin{array}{c}711 \cdot 7(77 \cdot 9) \\
70.0(14 \cdot 1) \\
406 \cdot 1(109 \cdot 5) \\
256.7(55 \cdot 7) \\
326 \cdot 1(68 \cdot 3) \\
236.9(31.6) \\
101 \cdot 3(53 \cdot 3) \\
417 \cdot 1(92 \cdot 7) \\
351 \cdot 3(78 \cdot 6) \\
360.0(80.6)\end{array}$ & 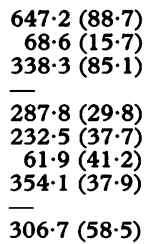 & $\begin{array}{l}0.002 \\
\text { NS } \\
0.016 \\
\overline{0.008} \\
\text { NS } \\
0.053 \\
0.007 \\
-0.031\end{array}$ \\
\hline
\end{tabular}

Abbreviations as in table 2 . slow-fast atrioventricular nodal echo was much more than in patients of group $A$ $(15 / 18,83.3 \%$ in group $B$, versus $6 / 31,19.4 \%$ in group $A, P$ \& 0.001$)$. The implicated site of ablation to the reentry circuit was probably twofold: the modification of the slow pathway component, and damage of the upper communicating path between the fast and the slow pathways. Particularly, the preferential damage of the latter alone might well result in the postablation electrophysiological profile of the group B patients: facilitated fast pathway, persistent slow pathway, inducible non-conducted atrioventricular nodal reentrant echo, but no sustained reentrant tachycardia.

\section{Selective fast pathway modification}

With the guidance of perinodal slow potential, inadvertent fast pathway ablation occurred in six of the 55 patients $(10.9 \%)$ (group C; C in fig 3): four with antegrade and retrograde fast pathway damage, two with selective retrograde fast pathway elimination. One of the latter two patients had had simultaneous eradication of the slow pathway, as shown by the loss of the discontinuity of the antegrade atrioventricular nodal conduction. None developed complete atrioventricular block from the first ablation procedure. The corresponding ablation sites for the inadvertent fast pathway damage were located at the anteroseptal zone in one patient, in the medioseptal zone in three patients, and in the posteroseptal zone in one patient.

FOLLOW UP ELECTROPHYSIOLOGICAL STUDY Three months after the success of catheter ablation, 40 patients $(72 \cdot 7 \%)$ agreed to have a follow up electrophysiological study (table 4): 23 from group $A$ (10 from subgroup A1, 13 from subgroup A2), 13 from group $B$, and four from group $C$. Under the same study protocol, including isoprenaline facilitation, three patients $(3 / 40,7 \cdot 5 \%)$ were shown to have reinducible sustained slow-fast atrioventricular nodal reentrant tachycardia. Among the three patients, two had clinical recurrence of the tachycardia. All three patients had been shown to have the eradication of atrioventricular nodal conduction duality early after the 
Table 4 Late phase electrophysiological data in 40 follow up patients. Values are means (SD), $m s$

\begin{tabular}{|c|c|c|c|c|}
\hline & Group $A 1(n=10)$ & $A 2(n=13)$ & $B(n=13)$ & $C(n=4)$ \\
\hline $\begin{array}{l}\text { Sinus CL } \\
\text { A-H } \\
\text { AVNERP } \\
\text { FPERP } \\
\text { AVBCL } \\
\text { 1:1 VA }\end{array}$ & $\begin{array}{c}866.0(156.0) \\
80.0(13.3) \\
443.0(201.9) \\
457.0(189.6) \\
497.0(180.9) \\
472.2(135.8)\end{array}$ & $\begin{array}{c}848 \cdot 5(107 \cdot 6) \\
85 \cdot 4(43 \cdot 9) \\
346 \cdot 2(99 \cdot 5) \\
365 \cdot 4(92 \cdot 6) \\
420 \cdot 8(101 \cdot 3) \\
382 \cdot 3(84 \cdot 5)\end{array}$ & $\begin{array}{c}839.2(100 \cdot 5) \\
75.0(12 \cdot 2) \\
374.6(64 \cdot 8) \\
406.9(71 \cdot 2) \\
464.6(90 \cdot 1) \\
396.2(82 \cdot 6)\end{array}$ & $\begin{array}{l}890 \cdot 0(40.8) \\
172.5(49.9) \\
345.0(183.4) \\
-\quad 47.5(128 \cdot 2) \\
580,460\end{array}$ \\
\hline
\end{tabular}

AVNERP, effective refractory period of atrioventricular node; AVBCL, blocking cycle length of 1:1 atrioventricular nodal conduction; other abbreviations as in table 2 .

previous catheter ablation: two from subgroup A1, one from subgroup A2. Analysis of the follow up electrophysiological data showed that return of the physiology of dual atrioventricular nodal pathways was more common than its disappearance: 13 of the 23 patients initially belonging to group A were reclassified as group $B$, while only three of 13 patients from group B converted to group A. One patient from the initial subgroup A1 had late disappearance of retrograde fast pathway conduction. Two of the three patients with initial bidirectional fast pathway modification remained the same, one with weak return of retrograde ventriculoatrial conduction. One patient with initially pure retrograde fast pathway damage had had late antegrade slow pathway modification on follow up. A single non-conducted or conducted atrioventricular nodal echo was inducible in 14 of 21 patients with antegrade atrioventricular nodal duality and no sustained tachycardia. None of 16 patients with eradication of the antegrade slow pathway or retrograde fast pathway had an inducible echo. The effective refractory period and the 1:1 conducting cycle length of the atrioventricular node were generally prolonged, supporting the progressive modification (but not eradication) of the slow pathway conduction as the main electrophysiological mechanism for the long term effectiveness.

The three patients with recurrent tachycardia received a second ablation procedure, also guided by the perinodal slow potential. Two were successfully reablated of the slow pathway and the tachycardia. The third patient unfortunately developed complete atrioventricular block, needing permanent pacemaker implantation. The ablation energy (30 W for only $10 \mathrm{~s}$ ) was focused at a medioseptal site with clear recording of the slow potential. The use of the perinodal slow potential as a local landmark resulted overall in $13.8 \%$ inadvertent fast pathway damage $(8 / 58)$, including $1.7 \%$ complete atrioventricular block (1/58).

\section{Discussion}

The present study confirmed the high therapeutic efficacy of transcatheter radiofrequency ablation in the eradication of slow-fast atrioventricular nodal reentrant tachycardia. However, the mere recording of perinodal slow potenial did not appear to be a guaranted of the success of the procedure. Onty $64.6 \%$ and $58.4 \%$ of the slow potential recording sites from the Koch's triangle had subse- quently resulted in the successful elimination of the reentrant tachycardia on the first and overall ablation attempts. Furthermore, the electrophysiological mechanisms of the resultant clinical success were actually very complex. Only $56.4 \%$ of the patients studied had the expected selective slow pathway eradication under the guidance of the perinodal slow potential. For the others, the combined damage to the upper communicating path and the slow pathway component of the reentry circuit $(32 \cdot 7 \%)$, or even inadvertent damage of the fast pathway $(10.9 \%)$, were thought to be the responsible mechanisms. A single local electrical landmark for specific slow pathway ablation does not seem to exist. Other than the recording of appropriately timed perinodal slow potential, the present study has actually recommended two concomitant characteristics common to a success site of catheter ablation, that is, the medioseptal location and the heating junctional tachycardia.

\section{PERINODAL SLOW POTENTIAL AND THE SLOW} PATHWAY

By contact mapping of high density plaque electrodes in human hearts, McGuire et $a \bar{l}$ have recently documented the existence of low frequency potentials between the atrioventricular node and the coronary sinus in Koch's triangle. Further correlative study by simultaneous extracellular and intracellular potential recordings from the same investigators suggests that the nodal type cells distributing subendocardially near the tricuspid annular border are the responsible tissue component of the slow potential with decremental conduction property. These observations confirm the single catheter finding of the same potential by Damato et $a l^{12}$ in 1969 and Haissaguerre et $a l^{4}$ in 1992. However, the functional correlation of the slow potential recording tissue to the slow pathway component of atrioventricular nodal reentry circuit remains largely speculative. The exact histological differentiation of the origin of the slow potential between atrionodal transitional cell, ${ }^{13}$ dead end fibres, ${ }^{14}$ and even the atrioventricular node ${ }^{1315}$ itself is also lacking.

As expected, success in the utilisation of the perinodal slow potential as a local landmark for transcatheter radiofrequency ablation of atrioventricular nodal reentrant tachycardia in the present study was also the result of complex mechanisms. The heterogeneous results reflect the heterogeneous sources of the slow potentials, and the corresponding tissue components in the region where the atrioventricular nodal reentry is located. The slow potential recorded in the Koch's triangle is apparently not a specific indicator of selective slow pathway ablation by radiofrequency current. Up to the present, the achievement of complication-free slow pathway elimination by catheter ablation still require cembined knowledge of the local anatomy, the slow conduction zone evaluation, for example, the' slow potential area, ${ }^{45} ?$ the junctional tachycardia during energy delivery, ${ }^{19}$ and so on. 
FAST PATHWAY FACILITATION AND

NON-CONDUCTED NODAL ECHO

In our study, the facilitation of fast pathway conduction was manifested in most patients after the successful catheter ablation of the atrioventricular nodal reentrant tachycardia. In contrast to other studies, ${ }^{320}$ we demonstrated that the shortening of effective refractory period and 1:1 conducting cycle length of the fast pathway would occur whether or not the slow pathway was totally eliminated.

Partly as a result of a concomitant decrease in the sinus cycle length, ${ }^{3421}$ the mechanism of the fast pathway facilitation was usually attributed to the postablation heightening of sympathetic tone or to the withdrawal of parasympathetic balance. ${ }^{22}$ However, recent studies $^{2023}$ with chemical autonomic blockade disputed the role of autonomic tone, and suggested that electrotonus interaction between the fast pathway and the slow pathway might rather be the determining factor. If the latter is correct, the ablation site in patients with postablation atrioventricular nodal duality (group B) in the present study must have involved the intervening atrial myocardium or the communicating fibres between the two pathways, particularly at the proximal portion. This is also compatible with the observation of more frequent induction of single, non-conducted slow-fast atrioventricular nodal reentrant echo in patients with residual slow pathway than those without it, early and late after the catheter ablation. The existence of non-conducted atrioventricular nodal reentrant echo theoretically implicates a missing link between the retrograde fast pathway and the antegrade slow pathway in the circuit of reentry, that is, the upper communicating path. A combination of the findings of fast pathway facilitation and non-conducted nodal reentrant echo in these patients strongly suggests strategic damage to the proximal communicating structure between the fast pathway and the slow pathway components of the reentry circuit.

\section{FOLLOW UP}

Long term electrophysiological follow up in the present study showed increasing reappearance of the slow atrioventricular nodal pathway. Non-conducted atrioventricular nodal reentrant echo, but never sustained tachycardia, remained easily inducible in those with residual dual atrioventricular nodal pathways. Persistent block of the upper communicating path is apparently at least as important as progressive modification of the slow pathway in preventing the recurrence of the previous reentrant tachycardia.

\section{LIMITATIONS}

First, discriminating the postablation electrophysiological mechanisms contributing to success of the procedure relied heavily on analysis of the atrioventricular nodad conduction curve, derived by classical extrastimulation or incremental pacing. Studies have provided firm evidence that dual atrioventricular nodal pathways could exist with various conduction patterns, with or without a jump of atrio-Hisian intervals. ${ }^{24} 25$ However, an accepted and standardised electrophysiological study with better evaluation of the atrioventricular nodal physiology is still pending in clinical practice. Second, the target mapping performed strictly along the tricuspid border of the Koch's triangle might have missed better sites locating away from the annulus. ${ }^{5}$ Technically, a more thorough search of the slow potential in the Koch's triangle is definitely feasible. However, the costbenefit balance of ablation over the atrial side of the tricuspid annulus and the risk of complete atrioventricular block has to be carefully evaluated. Third, the lack of temperature monitoring in radiofrequency energy delivery $^{26}$ might lead to misjudgment on the effect of an ablation attempt. The guiding accuracy of the perinodal slow potential could have been underestimated. A prospective study incorporating a thermometer tipped ablation catheter would be needed to answer this question.

\section{CONCLUSION}

This prospective study has shown the inadequacy of the perinodal slow potential in guiding selective slow pathway ablation of slow-fast atrioventricular nodal reentrant tachycardia by transcatheter radiofrequency current. The underlying electrophysiological mechanisms of the high clinical efficacy of this procedure involve selective eradication of the slow pathway, the intervening upper communicating path, or even the fast pathway. Careful evaluation of the local anatomy, heating junctional tachycardia, and the atrioventricular conduction status, in addition to the mapping of perinodal slow potential, is important for complication-free curative treatment of the atrioventricular nodal reentrant tachycardia. A specific and reliable electrical landmark for the slow pathway location in the Koch's triangle remains unknown.

The authors thank Miss Ying-Hwa Lin for the preparation of the manuscript.

1 Ross DL, Hohnson DC, Denniss AR, Cooper MJ, Richards DA, Uther JB. Curative surgery for atrioventricular junctional ("AV nodal") reentrant tachycardia. f Am Coll Cardiol 1985;6:1383-92.

2 Cox JL, Holman WL, Cain ME. Cryosurgical treatment of atrioventricular node reentrant tachycardia. Circulation atrioventricular node

3 Jackman WM, Beckman KJ, McClelland JH, Wang X-Z, Friday KJ, Roman CA, et al. Treatment of supraventricular tachycardia due to atrioventricular nodal reentry by radiofrequency catheter ablation of slow-pathway conduction. N Engl f Med 1992;327:313-8.

4 Haissaguerre $M$, Gaita $F$, Fischer $B$, Commenges D, Montserrat $\mathrm{P}$, d'Ivernois $\mathrm{C}$, et al. Elimination of atrioventricular nodal reentrant tachycardia using discrete slow potentials to guide application of radiofrequency energy. Circulation 1992;85:2162-75.

5 McGuire MA, Bourke JP, Robotin MC, Johnson DC, Meldrum-Hanna W, Nunn GR, et al. High resolution mapping of Koch's triangle using sixty electrodes in humans with atriouentricular junctional (AV nodal) humans with atrioventricular junctional (AV nodal) 2315-28.

6 McGuire MA, de Balter JMT, Vermetulen JT, Opthof T, Becker $\mathrm{AE}$, Jance MI. Origin and rignifcance of double potentials near the atrioventricular node: correlation of extracellular potentials, intracellular

7 de Bakker JMT, Coronel R, McGuire MA, Vermeulen JT, Opthof T, Tasseron S, et al. Slow potentials in 
atrioventricular junctional area of patients operated on for atrio-ventricular node tachycardia and in isolated for atrio-ventricular node tachycardia and in isol

8 Mazgalev T, Dreifus LS, Bianchi J, Michelson EL. The mechanism of AV junctional reentry: role of the atriomechanism of AV junctional reentry: role
nodal junction. Anat $\operatorname{Rec} 1981 ; 201: 179-88$.

9 Iinuma H, Dreifus LS, Mazgalev T, Price R, Michelson EL. Role of the perinodal region in atrioventricular nodal reentry: evidence in an isolated rabbit heart preparation. $\mathcal{F}$ Am Coll Cardiol 1983;2:465-73.

10 Wu D, Yeh S-J, Wang C-C, Wen M-S, Chang H-J, Lin F C. Nature of dual atrioventricular node path-ways and the tachycardia circuit as defined by radiofrequency ablation technique. $7 \mathrm{Am}$ Coll Cardiol 1992; 20:884-95.

11 Akhtar M, Jazayeri MR, Sra J, Blanck Z, Deshpande S, Dhala A. Atrioventricular nodal reentry: clinical, electrophysiological, and therapeutic considerations. Circulation physiological, and

12 Damato AN, Lau SH, Berkowitz WD, Rosen KM, Lisi KR. Recordings of specialized conducting fibers (AV nodal, His bundle, and right bundle branch) in man nodal, His bundle, and right bundle branch) in man using an

13 Anderson RH, Janse MJ, van Capelle FJL, Billette J, Becker AE, Durrer D. A combined morphological and electrophysiological study of the atrioventricular node of the rabbit heart. Circ Res 1974;35:909-22.

14 van Capelle FJL, Janse MJ, Varghese PJ, Freud GE, Mater $C$, Durrer D. Spread of excitation in the atrioventricular node of isolated rabbit hearts studied by multiple microelectrode recordings. Circ Res 1972;31:602-16.

15 Paes de Carvalho A, de Almeida DF. Spread of activity through the atrioventricular node. Circ Res 1960;8: 801-9.

16 James TN. Morphology of the human atrioventricular node, with remarks pertinent to its electrophysiology. Am Heart $\mathcal{7}$ 1961;62:756-71.

17 Janse MJ, Anderson RH, McGuire MA, Ho SY. "AV nodal" reentry: Part I: "AV nodal" reentry revisited. $f$ Cardiovasc Electrophysiol 1993;4:561-72.
18 Lauer MR, Young C, Munsif A, Yu J, Kuo C-T, Liem LB, et al. Identification of patients at high risk of developing ct al. Identification of patients at high risk of developing complete atrioventricular block during ablation of slow
pathway conduction [abstr]. Circulation 1993;88 (part 2): pathway

19 Thakur RK, Klein GJ, Yee R, Stites HW. Junctional tachycardia: a useful marker during radiofrequency ablation for atrioventricular node reentrant tachycardia. f Am Coll Cardiol 1993;22:1706-10.

20 Natale A, Klein G, Yee R, Thakur R. Shortening of the fast pathway refractoriness after slow pathway ablation: effects of autonomic blockade. Circulation 1994;89: 1103-8.

21 Ehlert FA, Goldberger JJ, Brooks R, Miller S, Kadish AH. Persistent inappropriate sinus tachycardia after radiofrequency current catheter modification of the atrioventricular node. Am $₹$ Cardiol 1992;69:1092-5.

22 Kay GN, Epstein AE, Dailey SM, Plumb VJ. Selective radiofrequency ablation of the slow pathway for the radiofrequency ablation of the slow pathway for the dia: evidence for involvement of perinodal myocardium within the reentrant circuit. Circulation 1992;85: within the

23 Lesh MD, Gibb WH, Epstein LM. Electrotonic interaction between dual AV nodal pathways: evidence from RF ablation and a computer model [abstr]. Circulation 1992;86(suppl I):I-130.

24 Tchou P, Mazgalev T, Keim S, Mowrey K, Cheng YN, Rist $\mathrm{K}$, et al. Towards a new definition of dual atrioventricular nodal pathways [abstr]. Circulation 1993;88 [part 2]:I-202.

25 Tchou P, Mazgalev T, Mowrey K, Cheng YN, Rist K. Interaction of dual pathways producing complex atrioventricular nodal conduction curves [abstr]. Circulation 1993;88[part 2]:I-203.

26 Hindricks G, Haverkamp W, Gulker H, Rissel U, Budde $\mathrm{T}$, Richter $\mathrm{KD}$, et al. Radiofrequency coagulation of ventricular myocardium: improved prediction of lesion size by monitoring catheter tip temperature. Eur Heart $\mathcal{f}$ 1989;10:972-84 